\title{
Nonlinear Transition from Mitigation to Suppression of the Edge Localized Mode with Resonant Magnetic Perturbations in the EAST Tokamak
}

\author{
Y. Sun, ${ }^{1}$ Y. Liang, ${ }^{1,2}$ Y. Q. Liu, ${ }^{3}$ S. Gu, ${ }^{1}$ X. Yang, ${ }^{4}$ W. Guo, ${ }^{1}$ T. Shi,,${ }^{1}$ M. Jia, ${ }^{1}$ L. Wang, ${ }^{1}$ B. Lyu,,${ }^{1}$ C. Zhou, ${ }^{5}$ A. Liu, ${ }^{5}$ Q. Zang, ${ }^{1}$ \\ H. Liu, ${ }^{1}$ N. Chu, ${ }_{1}^{1}$ H. H. Wang, ${ }^{1}$ T. Zhang, ${ }^{1}$ J. Qian, ${ }^{1}$ L. Xu, ${ }^{1}$ K. He, ${ }^{1}$ D. Chen, ${ }^{1}$ B. Shen, ${ }^{1}$ X. Gong, ${ }^{1}$ X. Ji, ${ }^{1}$ S. Wang, ${ }^{1}$ M. Qi ${ }^{1}$ \\ Y. Song, ${ }^{1}$ Q. Yuan, ${ }^{1}$ Z. Sheng, ${ }^{1}$ G. Gao, ${ }^{1}$ P. Fu, ${ }^{1}$ and B. Wan ${ }^{1, *}$ \\ ${ }^{1}$ Institute of Plasma Physics, Chinese Academy of Sciences, PO Box 1126, Hefei 230031, China \\ ${ }^{2}$ Institute for Energy and Climate Research-Plasma Physics, Forschungszentrum Jülich, 52425 Jülich, Germany \\ ${ }^{3}$ CCFE Culham Science Centre, Abingdon, OX14 3DB, United Kingdom \\ ${ }^{4}$ School of Physics and Optoelectronic Engineering, Dalian University of Technology, Dalian 116024, China \\ ${ }^{5}$ Department of Modern Physics, University of Science and Technology of China, Hefei 230026, China \\ (Received 1 February 2016; revised manuscript received 26 May 2016; published 9 September 2016)
}

\begin{abstract}
Evidence of a nonlinear transition from mitigation to suppression of the edge localized mode (ELM) by using resonant magnetic perturbations (RMPs) in the EAST tokamak is presented. This is the first demonstration of ELM suppression with RMPs in slowly rotating plasmas with dominant radio-frequency wave heating. Changes of edge magnetic topology after the transition are indicated by a gradual phase shift in the plasma response field from a linear magneto hydro dynamics modeling result to a vacuum one and a sudden increase of three-dimensional particle flux to the divertor. The transition threshold depends on the spectrum of RMPs and plasma rotation as well as perturbation amplitude. This means that edge topological changes resulting from nonlinear plasma response plays a key role in the suppression of ELM with RMPs.
\end{abstract}

DOI: 10.1103/PhysRevLett.117.115001

Magnetic reconnection and the resultant topological change play an important role in plasma dynamics in both laboratory and space plasma physics research. The formation of an edge stochastic magnetic field caused by resonant magnetic perturbations (RMPs) is believed to be the reason for the suppression of periodic crash events near the plasma edge known as the edge localized mode (ELM) observed in the DIII-D tokamak [1]. The ELM causes transient heat loads to the plasma facing components and may degrade them on the next generation fusion device like ITER [2]. The reduction of free energy in the edge pressure gradient and current because of field stochasticity moves the plasma into a stable regime against the ELM [3]. This successful experiment motivated ELM control using RMPs in many other tokamaks [4-7]. However, the plasma response effect usually shields the external applied RMPs and may significantly reduce the magnetic field stochasticity [8-11], which makes this mechanism questionable. Different from topological change, the linear peelinglike magneto hydro dynamics (MHD) response has been found to play an important role in ELM control [12-14]. Nonlinear plasma response has been observed in the JET totamak[15]. The possible formation of a magnetic island near the plasma edge [16] with a toroidal Fourier mode number $n=1$ during ELM suppression by using $n=2$ RMP has been recently observed on DIII-D [17]. However, the key difference between ELM suppression and mitigation and the different roles of linear and nonlinear plasma response on ELM suppression are still not clear.

In this Letter, we report the first observation of full ELM suppression using low $n$ RMPs in slowly rotating plasmas with dominant radio-frequency (rf) wave heating, which is potentially important for the application of this method for a future fusion device. This is the first observation of full ELM suppression using RMPs in the medium plasma collisionality regime in EAST, and it expands beyond the previous observations of ELM suppression on DIII-D $[1,3]$ and KSTAR [7]. It is found that not only the formation of a magnetic island near the edge [17] but also a critical level of magnetic topological change, taking into account plasma response, play a key role in accessing final ELM suppression. This observation also reveals different roles of linear and nonlinear response effects on ELM suppression.

A flexible in-vessel RMPs coil system consisting of two arrays of $(2 \times 8=16)$ coils was installed in the low field side in the superconducting tokamak EAST in 2014 $[18,19]$. We have successfully achieved both mitigation and full suppression of the type-I ELM by using $n=1$ and 2 RMPs in slow rotating plasmas with dominant rf wave heating in the EAST tokamak.

Full ELM suppression by using $n=1$ RMPs is observed in pure rf additional heating plasmas in EAST, when the perturbation field strength exceeds a threshold. Figure 1 shows the ELM behavior during the slow ramping up of the $n=1$ RMPs coil current in EAST pulse 55274. The additional heating powers from lower hybrid current drive (LHCD) $P_{\mathrm{LHCD}}=3 \mathrm{MW}$ and ion cyclotron radio frequency (ICRF) $P_{\mathrm{ICRF}}=1.4 \mathrm{MW}$ keep constant. The toroidal rotation near the plasma center is very close to 0 $(<4 \mathrm{krad} / \mathrm{s})$ measured by $\mathrm{x}$-ray crystal image spectrometry (XCIS) [20]. The toroidal field strength $B_{T}=2.25 \mathrm{~T}$, the 


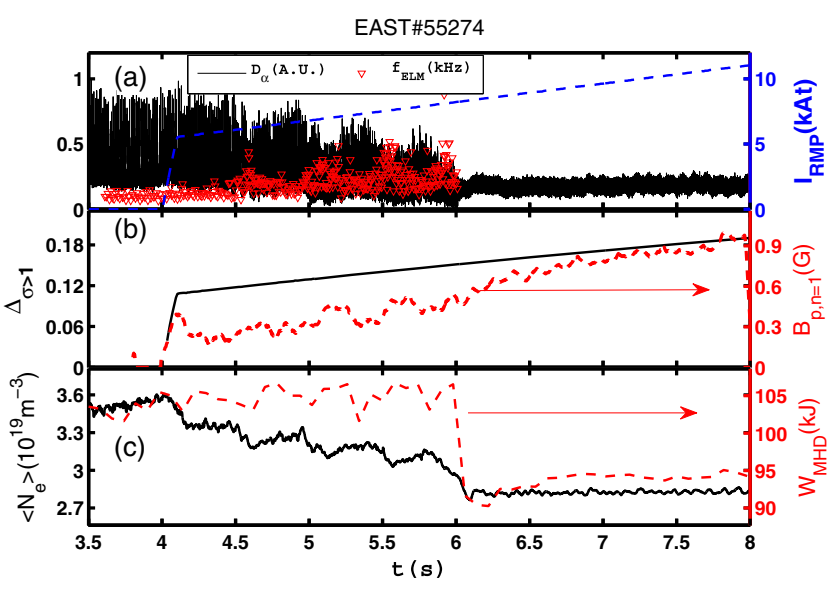

FIG. 1. Temporal evolution of (a) $D_{\alpha}$ (solid), ELM frequency (triangles) and $I_{\text {RMPs }}$ (dashed), (b) $\Delta_{\sigma>1}$ (solid) and the amplitude of the $n=1$ response poloidal magnetic field (dashed), (c) electron density (solid) and storied energy (dashed) for EAST pulse 55274.

safety factor at the surface with $95 \%$ of the normalized poloidal flux $q_{95} \approx 5.7$, the plasma current $I_{p}=0.45 \mathrm{MA}$ and normalized beta $\beta_{N} \approx 0.8$, and the normalized (to bounce or transit frequency of the particle orbit) collisionality near the pedestal top is around $\nu_{* e \text {,ped }} \sim 1$. As shown in Fig. 1, a stairlike decreasing of electron density and increasing of ELM frequency are observed during the application of RMPs before it ramps up to $8 \mathrm{kA}$ turns at $6 \mathrm{~s}$, after which the ELM is completely suppressed. The vacuum modeled width of the island overlapping area [19], $\Delta_{\sigma>1}=1-\left.\hat{\psi}_{p}^{1 / 2}\right|_{\sigma=1}$ (black solid line), is shown in Fig. 1(b). Here $\sigma$ is the Chirikov parameter indicating the condition of island overlapping and $\hat{\psi}_{p}$ is the normalized poloidal flux. The plasma responses at the ELM mitigation and suppression phases are obviously different. The amplitude of the $n=1$ perturbation field caused by the plasma response (red dashed line) measured by magnetic sensors is also shown in Fig. 1(b). The stairlike decreasing of electron density and increasing of ELM frequency before full suppression might be due to the penetration of different harmonics that have different penetration thresholds [21]. It suggests that the level of magnetic topological change, taking into account the plasma response, plays a key role in the final ELM suppression. This motivates us to further study the detailed evolution of the plasma response during the transition between ELM mitigation and suppression.

The transitions between ELM mitigation and suppression are observed by scanning of the phase difference between upper and lower coils $\delta \phi_{\mathrm{UL}}$, or equivalently the field strength of the resonant components. Figure 2 shows ELM control with a continuous scan of $\delta \phi_{\mathrm{UL}}$ [red line in (b)] by rotating the lower coil current with a frequency $f=0.5 \mathrm{~Hz}$ and keeping the upper one static with a constant amplitude $I_{\mathrm{RMP}}=10 \mathrm{kA}$ turns [red dashed line in (a)] in shot 55272. The target plasma with an ELM frequency around $100 \mathrm{~Hz}$ is similar to that in shot 55274 except for a minor difference in the heating scheme; i.e., there is a $0.7 \mathrm{MW}$ countercurrent neutral beam injection (NBI) instead of ICRF. It is still a rf dominant heating plasma. The changes of electron density [solid line in Fig. 2(c)] and ELM frequency [triangles in Fig. 2(b)] are very reproducible in the two periods of the $\delta \phi_{\mathrm{UL}}$ scan as also shown in Figs. 4(a) and 4(b). There are obviously three phases. In the first phase (I), i.e., $t \in[4,4.3] \mathrm{s}$ and $[6,6.3] \mathrm{s}$ corresponding to $\delta \phi_{\mathrm{UL}} \in[0,50]^{\circ}$, strong density pump-out and the ELM mitigation with ELM frequency increased by a factor of 5-10. The ELM is completely suppressed in phase (II) during $t \in[4.3,4.7] \mathrm{s}$ and $[6.3,6.7] \mathrm{s}$ or $\delta \phi_{\mathrm{UL}} \in[50,120]^{\circ}$, after a further sudden drop of electron density. In the third phase (III) for the rest of $\delta \phi_{\mathrm{UL}} \in[120,360]^{\circ}$, both the density pump-out and ELM mitigation effect are quite weak and almost keep constant after a sudden transition from ELM suppression. The variations of electron density and temperature profiles are shown in Figs. 2(f) and 2(g), respectively. It is shown that the electron density decreases but temperature increases during the application of RMPs. The plasma energy confinement during ELM mitigation is slightly better (higher storied energy but lower density) than the
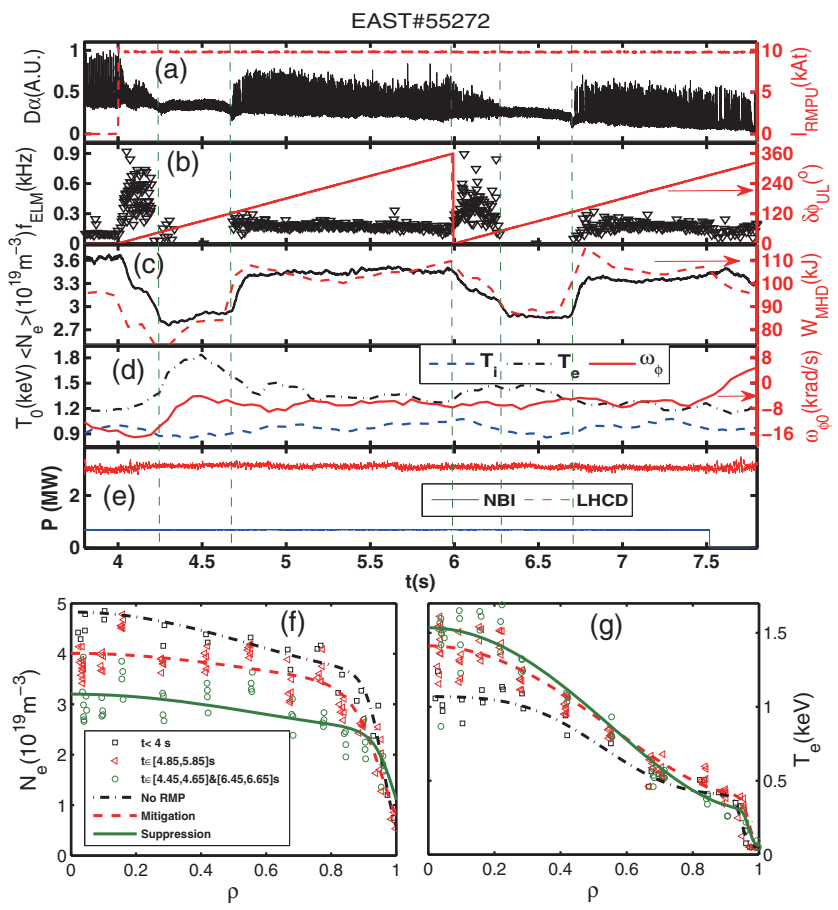

FIG. 2. Temporal evolution of (a) $D_{\alpha}$ (solid line) and the RMPs current $I_{\text {RMPs }}$ (dashed line), (b) ELM frequency (triangles) and $\delta \phi_{\mathrm{UL}}$ (solid line), (c) electron density (solid line) and storied energy (solid line), (d) electron (dashed-dotted line) and ion (dashed line) temperature and toroidal rotation (solid line) near the core, (e) NBI (solid line) and LHCD (dashed line) power, and the radial profiles of (f) electron density and (g) temperature during the periods without RMPs (dash-dotted line), mitigation (dashed line), and suppression (solid) for EAST pulse 55272. 


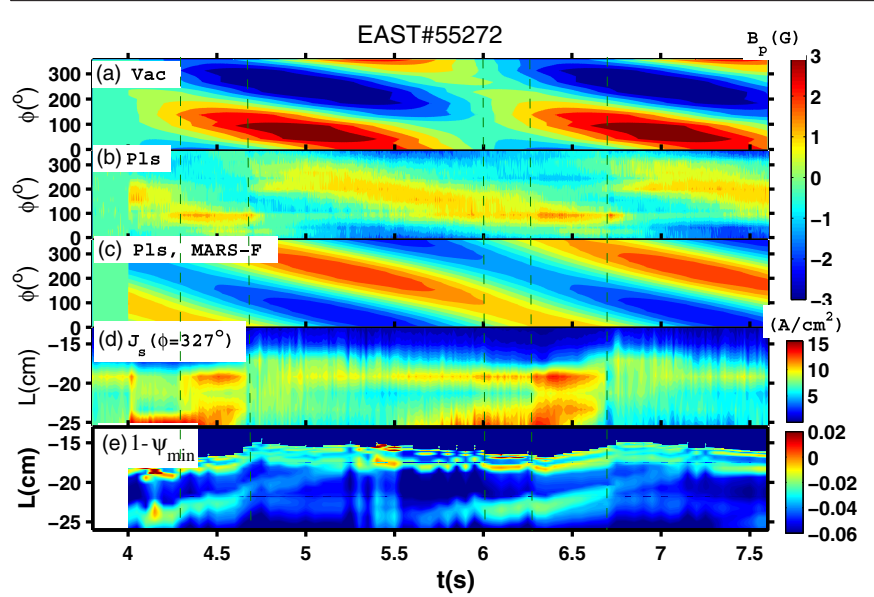

FIG. 3. (a)-(c) Contour plot of temporal evolution of the perturbation field (a) contributed from the RMPs vacuum field, (b) the measured plasma response, and (c) the plasma response modeled by the MARS-F code for a full toroidal array of magnetic sensors; (d) temporal evolution of the particle flux measured by one poloidal array of Langmuir probes on the upper divertor at $\phi=327^{\circ}$, and (e) the modeled deepest poloidal flux connected to the upper divertor for pulse 55272 .

period before the application of RMPs. Compared to ELM mitigation, full suppression gives a much stronger density pump-out effect and a slight drop of edge pedestal temperature. The energy confinement drops around $10 \%$ after the transition from ELM mitigation to full suppression.

The spectrum dependence of the ELM control effect suggests that total RMPs exceeding a critical value is a necessary condition for accessing ELM suppression. Similar to that observed in DIII-D, the best phase difference for ELM suppression is close to the resonant peak $\left(\approx 75^{\circ}\right)$ modeled by the linear MHD modeling code MARS-F [12], while it is different from the vacuum resonant peak $\left(\approx 356^{\circ}\right)$ modeled by MAPS [19]. However, the temporal evolution of plasma density is completely different from the observation on DIII-D [14], in which the density pump-out and magnetic braking almost changes like the cosine waveform during the $\delta \phi_{\mathrm{UL}}$ scan, indicating a linear plasma response in kinetic profiles.

Measurement shows clearly direct evidence of a nonlinear plasma response and the nonlinear transition or bifurcation between ELM mitigation and suppression in Figs. 3 and 4. The evolution of the observed plasma response field measured by the full toroidal array of poloidal magnetic sensors at the low field side is shown in Fig 3(b). The dominant component is the $n=1$ harmonic evaluated by using Fourier decomposition of the response field. The perturbation field from plasma response modeling by the MARS-F code shown in Fig. 3(c) well reproduces the general trend of the observations with a minor discrepancy. An equilibrium at $t=3.9 \mathrm{~s}$ of shot 55272 is used for the modeling shown here, because no significant change in the plasma response predictions was found by using a different equilibrium with or without RMPs ELM suppression in this discharge. However, the dependence of the phase of the $n=1$ response field on $\delta \phi_{\mathrm{UL}}$ shown in Fig. 4(c) illustrates a clear nonlinear transition between ELM mitigation and suppression. The measured phase of the $n=1$ response field follows well the linear MHD response one during the weak mitigation phase (III) with $\delta \phi_{\mathrm{UL}} \in[120,360]^{\circ}$, while it clearly deviates from the linear response one but is close to the vacuum one during the ELM suppression phase (II) with $\delta \phi_{\mathrm{UL}} \in[50,120]^{\circ}$. This suggests that the RMPs is shielded during ELM mitigation and the field penetration happens during the ELM suppression, because the penetrated resonant component has the same phase as the vacuum one while the shielding field has a phase shift to the vacuum one predicted in previous nonlinear modeling [22,23]. This means that the final ELM suppression requires field penetration, which cannot be described by the linear modeling. This may also explain a similar discrepancy between the measured response and MARS-F modeling found in DIII-D recently [24]. Different from the observation of field penetration on ELM suppression in DIII-D [17], the penetrated toroidal mode number here is the same as the applied one.

The phase of the response field gradually approaches the vacuum field one from ELM mitigation to full suppression as shown in Fig. 4(c), which suggests that different harmonics penetrate in turns and the degree of edge topological changes enhances gradually during this stage. The penetration threshold for different harmonics may be different [21]. Therefore, a possible reason is that there are multiple harmonics penetrated in turns during this transition. This explains also the stairlike changes of electron density and ELM frequency during the ramping up of the RMPs current before the final ELM suppression in shot 55274, shown in Fig. 1. Therefore, the degree of edge topological changes [3]

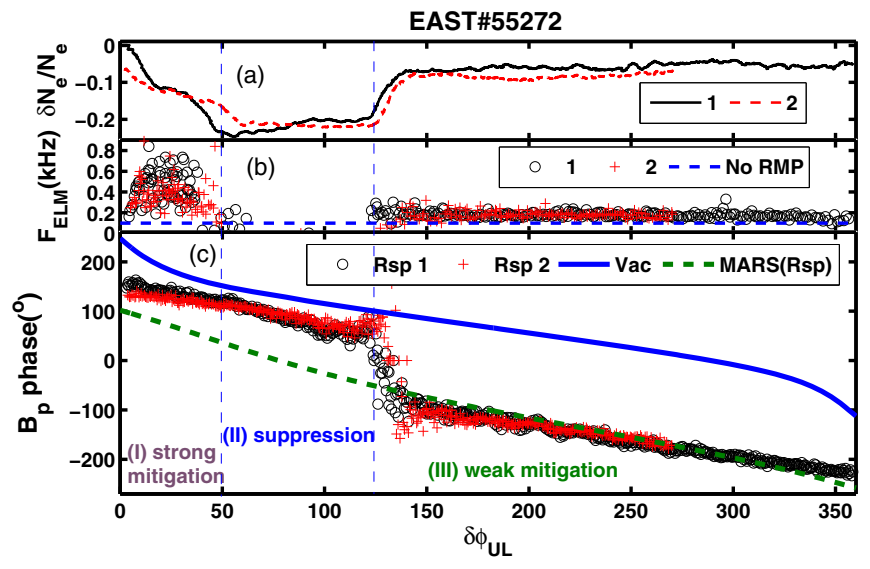

FIG. 4. Dependence of (a) plasma density, (b) ELM frequency, and (c) the phase of the $n=1$ response field from measurements (circles and pluses for the first and second periods) and modeling with vacuum field (solid line) and linear MHD response (dashed line) on $\delta \phi_{\mathrm{UL}}$ 
enhances gradually with the increasing amplitude of total RMPs field strength including plasma response and it results in the final ELM suppression. The sudden back transition from ELM suppression (II) to weak ELM mitigation (III) suggests that those resonant harmonics are shielded again almost simultaneously and the three-dimensional (3D) magnetic field structure disappears when the total RMPs field strength becomes lower than a threshold.

The observed footprint splitting of the particle flux to the divertor induced by RMPs during ELM suppression also is consistent with the edge topological changes. The 3D footprint was only observed when the plasma shielding effect decayed in $L$ mode plasmas on DIII-D [25]. As the RMPs field exceeded a threshold, the formation of a $3 \mathrm{D}$ footprint in both the particle and heat flux to the divertor is accompanied with a sudden increase of heat flux and drops of plasma density, indicating the formation of edge stochasticity, has been observed in $L$ mode plasmas on MAST [26]. The splitting pattern during strong ELM mitigation and suppression measured by one poloidal array of Langmuir probes on the upper divertor at $\phi=327^{\circ}$ shown in Fig. 3(d) is consistent with the vacuum modeling of the 3D footprint shown in Fig. 3(e) [27]. Significant enhancement of the particle flux also suggests field penetration and magnetic topological change during these phases, because this is a lower single null (LSN) configuration with the distance between the two separatrixes, $\left|d_{\text {rsep }}\right| \approx 1 \mathrm{~cm}$. This again supports the changes of edge topology during ELM suppression.

An additional sudden increase of edge perpendicular rotation is observed at the time when transition from ELM mitigation to full suppression occurs, which again supports the importance of edge topological changes on full suppression. Acceleration of the very edge rotation is evidence of the formation of edge stochasticity $[17,25,28]$. Figure 5 shows a comparison of ELM control effects between two shots, 56365 and 56366, with the same RMPs configuration and target plasmas with $B_{T}=1.7 \mathrm{~T}$, $I_{p}=0.45 \mathrm{MA}, \beta_{N} \approx 1.5$ and $q_{95} \approx 4.5$ except that the cocurrent NBI power of 56365 drops from $2 \mathrm{MW}$ to $1.2 \mathrm{MW}$ at $t \approx 3.6 \mathrm{~s}$ in Fig. 5(b). As shown in Fig. 5(a), a step rotating RMPs with phases $[315,225,135,45]^{\circ}$, keeping the constant current amplitude $I_{n=2}=14 \mathrm{kA}$ turns and $\delta \phi_{\mathrm{UL}}=270^{\circ}$, is applied for both of these two shots. Strong ELM mitigation with a factor of 5 increase in ELM frequency is observed, after the application of RMP in these two shots. Full ELM suppression is only achieved after an additional sudden increase of edge perpendicular rotation in shot 56365 at $t \approx 3.9 \mathrm{~s}$, after a slow decay of plasma rotation because of the reduction of NBI power at $3.6 \mathrm{~s}$. The Mirnov signal $d B / d t$ is used as a measure of the ELM crashes, because it is more sensitive to the small ELM crash during the strong mitigation. It is shown in Fig. 5(b) that there is a sudden increase of the very edge perpendicular rotation measured by the Doppler backscattering system (DBS) [29] immediately after the application of RMPs, which indicates the change of

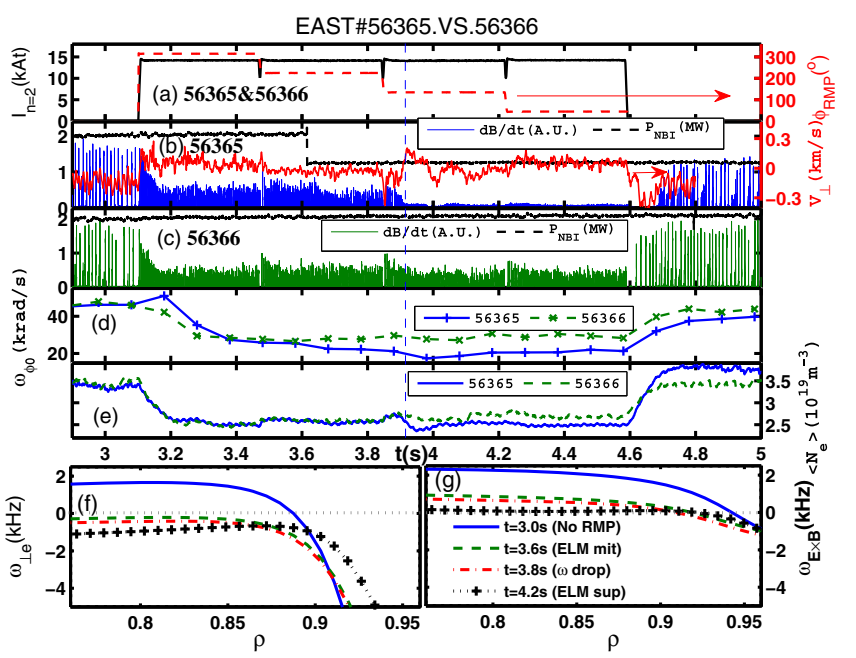

FIG. 5. Temporal evolution of (a) the amplitude (solid line) and phase (dashed line) of the $n=2$ RMPs current for EAST shots 56365 and 56366, (b) the $d B / d t$ (solid line), the NBI power (dashed line) and the edge perpendicular rotation (red) for shot 56365 , (c) the $d B / d t$ (solid line) and the NBI power (dashed line) for shot 56366, (d) the toroidal rotation frequency near the core, and (e) the electron density, and the radial profiles of (f) $\omega_{e \perp}$, and (g) $\omega_{E \times B}$, at $t=3.0 \mathrm{~s}, 3.6 \mathrm{~s}, 3.8 \mathrm{~s}$, and $4.2 \mathrm{~s}$ for shot 56365 .

edge topology [28]. As shown in Figs. 5(f) and 5(g), the evaluated electron fluid perpendicular rotation, $\omega_{e \perp}$, and $\vec{E} \times \vec{B}$ one, $\omega_{E \times B}$, near the pedestal top becomes very close to 0 after the application of RMPs because of rotation braking [30-32]. According to recent plasma response theories [11] and modelings $[8,10,22]$, the resonant harmonics near the pedestal top, where $\omega_{e \perp} \approx 0$, may penetrate. During ELM suppression, the $\omega_{e \perp}$ becomes closer to zero near the pedestal top at around $\rho=0.9$, and the $\omega_{E \times B}$ becomes completely flat inside $\rho=0.92$. Some more modes may penetrate, which results in the final ELM suppression. This suggests that the strong ELM mitigation is also linked to field penetration and edge topological change, and the final transition to ELM suppression requires that the edge topological change exceeds a critical level.

In summary, evidence of a nonlinear transition from mitigation to suppression of the ELM by using $n=1$ and 2 RMPs have been observed in the EAST tokamak. This first demonstration of ELM suppression with low $n$ RMPs in low rotating plasmas with dominant rf wave heating is potentially important for a future fusion device. The linear MHD modeling reveals the total RMPs field strength, including the plasma contribution rather than the vacuum one, determines the optimized coil configuration for full ELM suppression. The phase of response field gradually deviates the linear MHD result and approaches the vacuum field one during the transition from mitigation to suppression. This suggests that different harmonics penetrate in turns and the degree of edge topological changes enhances gradually during this stage.. It explains also the observed 
stairlike changes of electron density and ELM frequency during the ramping up of the RMPs current before the final ELM suppression. The footprint splitting and the sudden increase of particle flux to the divertor also support the edge topological change during ELM suppression. An additional sudden increase of edge perpendicular rotation triggers the transition from ELM mitigation to full suppression and it suggests the existence of a threshold in edge topological change for full suppression. Therefore, not only the formation of a magnetic island near the edge, but also a critical level of magnetic topological change taking into account plasma response, play a key role in accessing final ELM suppression. However, modeling of the nonlinear plasma response to RMPs is still a big challenge [33]. In future studies, more efforts should be made in the understanding of nonlinear plasma response, especially the key issues for the transition or bifurcation..

This work is supported by the National Natural Science Foundation of China under Grants No. 11475224 and No. 11205199, and the National Magnetic Confinement Fusion Science Program of China under Grants No. 2013 GB102000, No. 2013 GB106003B, and No. 2012 GB105000.

*bnwan@ipp.ac.cn

[1] T. Evans et al., Phys. Rev. Lett. 92, 235003 (2004).

[2] R. J. Hawryluk et al., Nucl. Fusion 49, 065012 (2009).

[3] T. E. Evans et al., Nat. Phys. 2, 419 (2006).

[4] Y. Liang et al., Phys. Rev. Lett. 98, 265004 (2007).

[5] A. Kirk et al., Nucl. Fusion 50, 034008 (2010).

[6] W. Suttrop et al., Phys. Rev. Lett. 106, 225004 (2011).

[7] Y. M. Jeon et al., Phys. Rev. Lett. 109, 035004 (2012).

[8] M. F. Heyn, I. B Ivanov, S. V Kasilov, and W. Kernbichler, Nucl. Fusion 46, S159 (2006).

[9] Y. Liu, A. Kirk, and E. Nardon, Phys. Plasmas 17, 122502 (2010).
[10] M. Becoulet et al., Nucl. Fusion 52, 054003 (2012).

[11] F. L. Waelbroeck, I. Joseph, E. Nardon, M. Bécoulet, and R. Fitzpatrick, Nucl. Fusion 52, 074004 (2012).

[12] Y. Liu, A. Kirk, Y. Gribov, M. P. Gryaznevich, T. C. Hender, and E. Nardon, Nucl. Fusion 51, 083002 (2011).

[13] A. Kirk et al., Nucl. Fusion 55, 043011 (2015).

[14] C. Paz-Soldan et al., Phys. Rev. Lett. 114, 105001 (2015).

[15] Y. Liang et al., Nucl. Fusion 53, 073036 (2013).

[16] P. Snyder, T. H. Osborne, K. H. Burrell, R. J. Groebner, A. W. Leonard, R. Nazikian, D. M. Orlov, O. Schmitz, M. R. Wade, and H. R. Wilson, Phys. Plasmas 19, 056115 (2012).

[17] R. Nazikian et al., Phys. Rev. Lett. 114, 105002 (2015).

[18] B. Wan, J. Li, H. Guo, Y. Liang, and G. Xu et al., Nucl. Fusion 53, 104006 (2013).

[19] Y. Sun, Y. Liang, J. Qian, B. Shen, and B. Wan, Plasma Phys. Controlled Fusion 57, 045003 (2015).

[20] B. Lyu et al., Rev. Sci. Instrum. 85, $11 E 406$ (2014).

[21] D. Reiser and D. Chandra, Phys. Plasmas 16, 042317 (2009).

[22] Q. Yu, S. Günter, Y. Kikuchi, and K. H. Finken, Nucl. Fusion 48, 024007 (2008).

[23] H. Reimerdes et al., Nucl. Fusion 49, 115001 (2009).

[24] S. R. Haskey, M. J. Lanctot, Y. Q. Liu, C. Paz-Soldan, J. D King, B. D Blackwell, and O. Schmitz, Plasma Phys. Controlled Fusion 57, 025015 (2015).

[25] O. Schmitz, T. E. Evans, M. E. Fenstermacher, M. J. Lanctot, C. L. Lasnier, S. Mordijck, R. A. Moyer, and H. Reimerdes, Nucl. Fusion 54, 012001 (2014).

[26] A. J. Thornton, A. Kirk, P. Cahyna, I. T. Chapman, J. R. Harrison, and Y. Liu, Nucl. Fusion 54, 064011 (2014).

[27] M. Jia, Y. Sun, F. Zhong, H. Li, G. Li, L. Wang, K. Gan, B Zhang, J. Qian, and B. Shen, Plasma Phys. Controlled Fusion 58, 055010 (2016).

[28] K. H. Finken et al., Phys. Rev. Lett. 94, 015003 (2005).

[29] C. Zhou et al., Rev. Sci. Instrum. 84, 103511 (2013).

[30] K. C. Shaing, Phys. Plasmas 10, 1443 (2003).

[31] W. Zhu et al., Phys. Rev. Lett. 96, 225002 (2006).

[32] Y. Sun et al., Nucl. Fusion 52, 083007 (2012).

[33] A. D. Turnbull et al., Phys. Plasmas 20, 056114 (2013). 